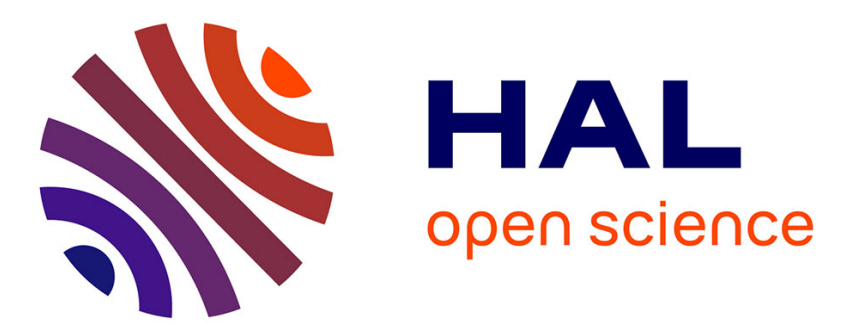

\title{
Analysis of Head Losses in a Turbine Draft Tube by Means of 3D Unsteady Simulations
} Sylvia Wilhelm, Guillaume Balarac, Olivier Métais, Claire Ségoufin

\section{To cite this version:}

Sylvia Wilhelm, Guillaume Balarac, Olivier Métais, Claire Ségoufin. Analysis of Head Losses in a Turbine Draft Tube by Means of 3D Unsteady Simulations. Flow, Turbulence and Combustion, 2016, 97 (4), pp.1255-1280. 10.1007/s10494-016-9767-9 . hal-01418777

\section{HAL Id: hal-01418777 \\ https://hal.science/hal-01418777}

Submitted on 17 Apr 2019

HAL is a multi-disciplinary open access archive for the deposit and dissemination of scientific research documents, whether they are published or not. The documents may come from teaching and research institutions in France or abroad, or from public or private research centers.
L'archive ouverte pluridisciplinaire $\mathbf{H A L}$, est destinée au dépôt et à la diffusion de documents scientifiques de niveau recherche, publiés ou non, émanant des établissements d'enseignement et de recherche français ou étrangers, des laboratoires publics ou privés. 


\title{
Analysis of head losses in a turbine draft tube by means of $3 \mathrm{D}$ unsteady simulations
}

\author{
Sylvia Wilhelm · Guillaume Balarac . \\ Olivier Métais · Claire Ségoufin
}

Received: date / Accepted: date

\begin{abstract}
In this paper, Unsteady RANS (URANS) simulations and Large Eddy Simulations (LES) in the draft tube of a bulb turbine are presented with the objective to understand and locate the head losses in this turbine component. Three operating points of the turbine are considered. Numerical results are compared with experimental velocity measurements for validation. Thanks to a detailed analysis of the energy balance in the draft tube, the physical and hydrodynamic phenomena responsible for head losses in the draft tube are identified. Head losses are due to transfer of mean kinetic energy to the turbulent flow and viscous dissipation of kinetic energy. This occurs mainly in the central vortex structure and next to the walls in the draft tube. Head losses prediction is found to be dependent on the turbulence model used in the simulations, especially in URANS simulations. Using this analysis, the evolution of head losses between the three operating points is understood.
\end{abstract}

Keywords Unsteady RANS · Large Eddy Simulation · turbulence · hydraulic turbine $\cdot$ draft tube $\cdot$ head losses

\section{Introduction}

Hydroelectric energy production by exploitation of low head sites is an attractive alternative to the increase of energy demand. Bulb turbines constitute the most efficient solution for low head sites thanks to their horizontal axis which enables low construction costs and high performances [1]. Moreover, large operating ranges are ensured by these double regulated turbines: guide vanes and blades angles are both varying in order to reach optimal performances.

The performances of low head turbines are highly influenced by the head losses in the draft tube. The draft tube has a divergent shape in order to convert the residual kinetic energy leaving the runner into pressure and thus increase the effective head

S. Wilhelm · G. Balarac · O.Métais

Grenoble-INP/CNRS/UJF-Grenoble 1, LEGI UMR 5519, Grenoble, F-38041, France

E-mail: Sylvia.Wilhelm@legi.grenoble-inp.fr

C. Ségoufin

GE Renewable Energy, Hydro, 82 avenue Léon Blum, Grenoble, 38041, France 
of the turbine [2]. For low head turbines, the flow in the draft tube can lead to high energy losses in comparison with other turbine components. The prediction of head losses in the draft tube of bulb turbines is thereby a major issue. This can be done by means of experiments on test platform or by means of numerical simulation which is less expensive, more flexible and leads to a more complete database on the flow field. A better understanding of the flow phenomena occurring in the draft tube could thus be achieved with high fidelity numerical simulations. However, the complexity of the flow in the draft tube (highly turbulent, swirling and decelerating) renders the numerical prediction of the head losses very challenging.

In industry, steady RANS simulations of the draft tube flow using two equations linear eddy-viscosity turbulence models are usually used due to their affordable computational cost. These models are robust, but the steady RANS approach is unable, by construction, to predict the unsteady flow in the draft tube [3]. Even with Unsteady RANS (URANS) simulations, unsteadiness and turbulent flow structures are damped out when linear eddy-viscosity turbulence models are used [4,5]. Moreover, two equations turbulence models are not able to correctly account for turbulent production due to streamline curvature in swirling flows [6]. Corrections of these models, such as curvature correction [6,7] or turbulent production limiter [8], can be used to improve the prediction of swirling flows in draft tubes [9]. Moreover, advanced RANS models such as Reynolds-stress-transport models, algebraic Reynolds-stress models, and non-linear eddy-viscosity models can lead to a better prediction of swirling flows since they are more sensitive to flow instabilities and unsteadiness. However, when using statistical approaches, the turbulent part of the flow field is always modelled and only the large mean scales of the flow are explicitly solved. A better understanding of the physic of highly turbulent flows in draft tubes can be obtained with improved turbulence models such as Scale Adaptive Simulations (SAS), Zonal Large Eddy Simulations (ZLES) or Hybrid RANS-LES turbulence models such as Detached Eddy Simulations (DES) $[10,4,11,12]$. In particular, DES in a Francis turbine draft tube were performed in $[4,11,12]$ which led to a more complete physical description of the flow than with URANS simulations. However, head losses prediction was not improved with DES simulations. Therefore an improvement of the flow prediction can be expected with LES which could capture the complex, 3D phenomena developing in the draft tube, but with a much higher computational cost than for classic RANS calculations [13]. Moreover, the authors of [11-13] point out that accurate inlet boundary conditions accounting for the unsteadiness from the runner are necessary to improve the flow prediction in the draft tube. The strong sensitivity to inlet velocity profiles prescription on the flow prediction in the draft tube of axial turbines has indeed been observed in several studies [14-18]. In particular, an accurate description of the flow from the hub and shroud gaps is necessary $[19,20]$.

The objective of this paper is to perform a precise analysis of the highly turbulent flow in the draft tube of a bulb turbine in order to better understand the flow phenomena leading to head losses in this component. Both Unsteady RANS (URANS) calculations and Large Eddy Simulations (LES) are used for this purpose and their reliability is evaluated through comparisons with experimental data. As previously pointed out, numerous URANS modelling approaches have been developed but we here restrict our study to URANS based on the $k-\omega$ SST turbulence model [21] since it is the most widely used in hydraulic machinery industry for its recognized performances $[22,9,17]$. In these numerical simulations, unsteady inlet boundary conditions are used to take into account the unsteady phenomena coming 
from the runner. From these simulations, a local analysis of the predicted flow field using the energy balance in the draft tube is performed to understand the main physical phenomena responsible for head losses. Three operating points are considered: the best efficiency point of the turbine, leading to the lowest head losses, and two off-design points.

\section{Methodology}

The computational domain is illustrated in figure 1 . We do not simulate here the complete system constituted of the turbine and the draft tube. The whole draft tube is computed with only the runner tip of the bulb turbine which rotates at the runner speed. A straight extension is added at the outlet of the draft tube in order to improve calculation convergence and to avoid influence of outlet boundary condition on the real outlet of the draft tube.

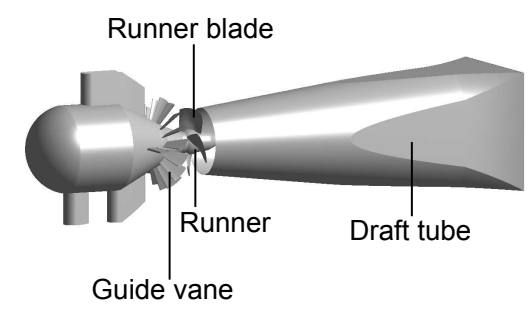

(a)

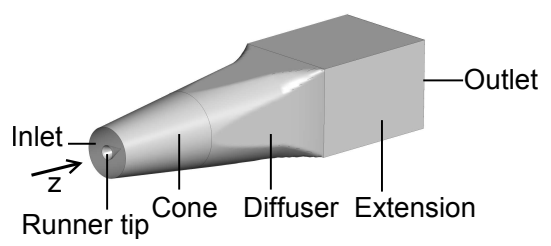

(b)

Fig. 1 Whole domain of a bulb turbine (a) and computational domain (b)

\subsection{Inlet boundary conditions}

The inlet velocity profiles for the draft tube simulations are extracted from the outlet of a RANS simulation of one guide vane - runner blade passage, performed by using the commercial flow solver ANSYS CFX. The computational domain for this RANS calculation is composed of one guide vane and one runner blade assuming periodicity of the flow. Periodic boundary conditions are applied to the guide vane and runner angular boundaries. A stage interface [23] is used to treat the rotor-stator interface between the guide vane domain (fixed domain) and the runner (rotating domain). This leads to circumferential averaging of fluxes at the interface. The guide vane runner blade simulation is conducted using the $k-\omega$ SST turbulence model on a hexahedral mesh composed on 2 million elements. The mass flow corresponding to the studied operating point is imposed at the inlet of the guide vane domain with a flow angle assuming that the flow is parallel to the guide vane passage. A turbulent intensity of $5 \%$ and a turbulent length scale of $10 \%$ of the inlet height are imposed at the inlet. Phase-averaged velocity profiles on the inlet plane of the draft tube, included in this calculation, are thus extracted and duplicated 3 times, since the runner contains 4 blades, to obtain the two-dimensional inlet profiles of the draft 
tube. In steady RANS simulations of the draft tube or in full-machine simulations using a stage averaging [23] between the runner and the draft tube, the flow is supposed to be totally mixed at the outlet of the runner. An azimuthal averaging is then performed to prescribe a steady axisymmetric velocity profile at the draft tube inlet (see figure 2a). This is a strong assumption for bulb turbines, as they have a small number of blades so that the field at the runner outlet is inhomogeneous in azimuthal direction between two successive blade passages [24]. In the present work, the velocity profiles are imposed at the inlet of the draft tube without any recourse to azimuthal averaging (see figure $2 \mathrm{~b}$ ). We impose a rotation of these profiles at the runner rotating speed to preserve the unsteadiness of the fields. In particular, hub and gap vortices and blade wakes are thus taken into account. This procedure is close to full-machine simulations using transient rotor-stator interface between the runner and the draft tube in which the true unsteady interaction between the runner and the draft tube is taken into account [23]. For simplicity, only velocity profiles are here prescribed. This enables us to conduct several URANS simulations and LES of the draft tube flow with only one RANS simulation of the guide vane - runner per operating point.

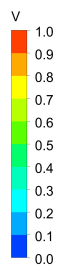

(a) Axisymmetric velocity profile

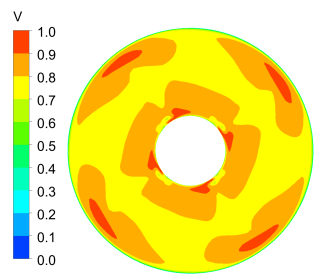

(b) $2 \mathrm{D}$ velocity profile

Fig. 2 Axisymmetric non-dimensional velocity profile (a) and two-dimensional velocity profile (b) at the draft tube inlet

\subsection{URANS simulations}

The commercial flow solver ANSYS CFX is used to perform URANS calculations. The $k-\omega$ SST turbulence model is here chosen since, as shown by several studies $[9,21,17]$, it appears to be the most suitable linear eddy-viscosity model for flows under adverse pressure gradient as in draft tubes. As far as numeric is concerned, the "high resolution scheme" of ANSYS CFX is used for the advection term. It is based on a blend factor, acting as a limiter from a first order upwind scheme to a second order central scheme to keep the solution bounded. For the two extra transport equations for $k$ and $\omega$, a first order scheme is used. Since we here deal with unsteady simulations, the temporal derivative of the Navier-Stokes equations is discretized using the second order backward Euler scheme. The temporal derivative for the transport equations for $k$ and $\omega$ is discretized using the first order backward 
Euler scheme. In $k-\omega$ SST URANS simulations, the prescription at the inlet of turbulence quantities is necessary to be able to resolve the transport equations for $k$ and $\omega$. In steady RANS simulations, uniform turbulent quantities are usually imposed according to best practice guidelines [17]. In the present work, $k$ and $\omega$ profiles are extracted from the guide vane - runner RANS simulation and imposed at the draft tube inlet similarly as for velocity profiles (see section 2.1 ). The averaged static pressure at the outlet is imposed equal to 0. No-slip condition is imposed on the stationary walls. The runner tip is a rotating wall with a velocity equal the runner rotation speed. Note that we have verified that these inlet conditions lead to the same results a fully coupled simulation including guide vane, runner and draft tube with transient rotor-stator interface between the runner and the draft tube. This indicates that the flow in the draft tube has very little influence on the runner flow field and both calculations can therefore be decoupled.

The block-structured hexahedral mesh for the draft tube is composed on 2 million elements (including the extension). The size of the first cells close to the wall in wall units, $y^{+}$, has a mean value of 5 and varies from 10 on the runner tip to 0.1 in the extension. A logarithmic wall function is used in CFX [25]. Mesh convergence has been checked for the draft tube to assess the independence of the results (see appendix A.1).

Convergence tests lead to a time step corresponding to five degrees of runner rotation per time step. The transient part of the simulations is performed for approximately four flow passages through the draft tube. Flow statistics are calculated on a time length corresponding to two flow passages through the draft tube which proved to be sufficient for statistical convergence. This methodology is an improvement of the classical RANS simulations of the draft tube because of the unsteadiness of the computation but also thanks to an improved description of the inlet velocity profiles.

\subsection{Large Eddy Simulations (LES)}

LES computations are carried out with the YALES2 incompressible fractional-step solver with a finite-volume formulation with numerical schemes of 4 th order in time and space [26]. The dynamic Smagorinsky subgrid-scale model is used [27].

No turbulence is imposed at the inlet. In this swirling flow, the instabilities are found to develop without the addition of inflow perturbations. As for the URANS computations, a rotating wall at the runner speed is imposed for the runner tip. No-slip condition is imposed on the walls.

The mesh is composed of tetrahedral elements with prisms layers at the wall in order to ensure a small value of $\mathrm{y}+$ with a reasonable number of elements. A mesh composed of 16 million elements with a $\mathrm{y}+$ ranging from 10 to 20 is generated. Analysis on the near-wall mesh resolution and on the discretization of the internal flow have shown that this resolution is enough for the flow prediction in the draft tube (see appendix A.2). The time step complies with the CFL condition and corresponds to around 0.07 degree of runner rotation. The simulation is run until quantities of interest are converged (head losses, velocity profiles). This transient stage needs between two and four flow passages through the draft tube, depending on the operating points. Flow statistics are then calculated. The statistics are computed on a period corresponding to four flow passages through the draft tube. 


\section{Flow prediction in the draft tube}

The flow field in the draft tube is calculated for three operating points of the bulb turbine, from low to high flow rate, with a constant blade angle. OP1 corresponds to the off-design point at partial load (low flow rate). OP2 corresponds to the optimal point, where the efficiency of the turbine is the highest. OP3 corresponds to the point at high load (high flow rate). Table 1 gives the non-dimensional parameters for each operating point. The Reynolds number is defined with the bulk velocity and the maximum radius on the inlet section. The Swirl number is defined as,

$$
S=\frac{1}{R_{2}-R_{1}} \frac{\int_{S} V_{z} V_{u} r d S}{\int_{S} V_{z}^{2} d S}
$$

where $V_{z}$ and $V_{u}$ are respectively the axial and tangential velocity, $r$ is the radius on the inlet section and $R_{1}$ and $R_{2}$ are respectively the minimum and maximum radius at the inlet section.

Table 1 Flow parameters for the considered operating points. $Q$ is the flow rate, $R e$ is the Reynolds number and $S$ is the Swirl number.

\begin{tabular}{cccc}
\hline Operating point & $Q / Q_{O P 2}$ & $R e$ & $S$ \\
\hline OP1 & 0.954 & $1.10^{6}$ & 0.34 \\
OP2 & 1.000 & $2.4 .10^{6}$ & 0.23 \\
OP3 & 1.122 & $2.7 .10^{6}$ & $-0.4 .10^{-3}$ \\
\hline
\end{tabular}

3.1 Validation of the simulations for the best efficiency point OP2

For the validation of the numerical simulations, experimental axial $\left(V_{z}\right)$ and tangential $\left(V_{u}\right)$ velocity profiles, measured by means of 2D-LDV (Laser Doppler Velocimetry), are used at three stations in the draft tube shown in figure 3: stations $\mathrm{A}$ and $\mathrm{B}$ in the cone and station $\mathrm{C}$ in the diffuser. The axial velocity $V_{z}$ is the velocity component along the $z$ axis (see figure 3 ) and the tangential velocity $V_{u}$ is positive in the runner rotation direction. Note that velocities presented in this paper are dimensionless, obtained by dividing velocity values by the bulk velocity at OP2 $V_{b, O P 2}$.

The predicted velocity profiles with LES and URANS calculations for the best efficiency point (OP2) are compared with the experimental profiles at the three measured stations in the draft tube in figure 4 . Here, $R_{\max }$ designates the maximum radius at station $C$. Velocity profiles obtained with URANS and LES are close in stations $\mathrm{A}$ and $\mathrm{B}$ but differ from experimental results. Indeed, the experimental velocity profiles at stations $\mathrm{A}$ and $\mathrm{B}$ show a low axial velocity, near the axis $\left(R / R_{\max }\right.$ going to zero), associated with an important increase of the tangential velocity from the center of the draft tube up to a normalized radius of 0.1 . This is a characteristic feature of the central vortex structure in the draft tube generated under the runner tip [28]. The axial velocity in the central zone is strongly overestimated by both the 


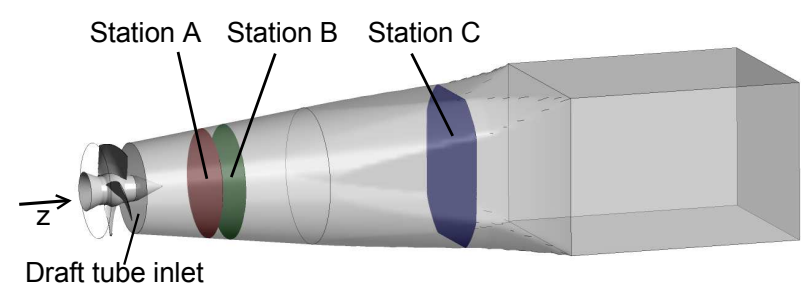

Fig. 3 Experimental measurement stations for velocity profiles

LES and URANS simulation. Moreover, the tangential velocity is underestimated by the simulations in the most part of the sections A and B. This indicates that the central vortex structure is poorly predicted by the calculations.

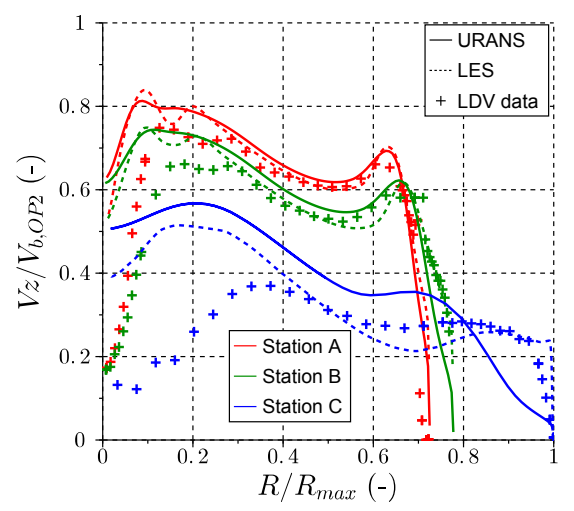

(a) Axial velocity

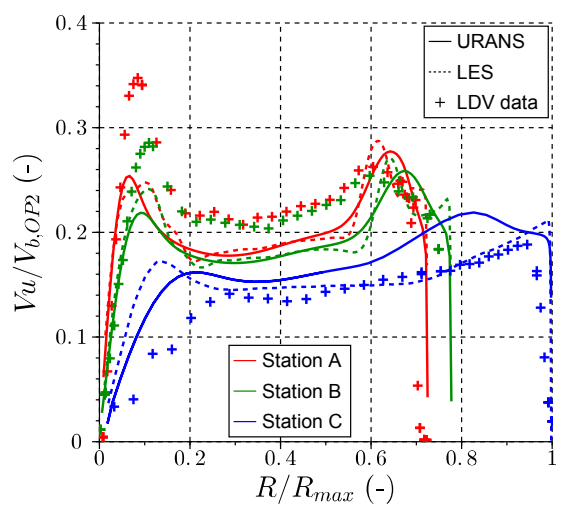

(b) Tangential velocity

Fig. 4 Non-dimensional axial (a) and tangential (b) velocity profiles ( $\mathrm{Vz}, \mathrm{Vu}$ ) versus the non-dimensional radial position for OP2

Since, for both calculations, the tangential velocity is underestimated at station A which is close to the inlet, one may reasonably suppose that the tangential velocity at the inlet itself is already lower than the real value. This indicates that the quality of the RANS simulation of the guide vane - runner used as inlet condition has to be improved. This will be done in further study. To obtain better inlet conditions in a simple way, we here choose to enhance the tangential velocity level at the draft tube inlet simply by increasing the turbine rotation speed $n$. A new RANS calculation is therefore conducted with rotation speed $n+2 \%$. The velocity profiles obtained at the draft tube inlet, with the original calculation with runner speed $n$ and the new calculation with runner speed $n+2 \%$ are shown in figure 5 . By increasing the runner rotation speed, the tangential velocity is increased at the inlet but the axial velocity remains almost unchanged, except near the hub $\left(R / R_{\max }\right.$ around 0.35$)$.

The velocity profiles corresponding to $n+2 \%$ are now used as inlet conditions for new URANS simulation and LES. The tangential velocity is thus increased at 


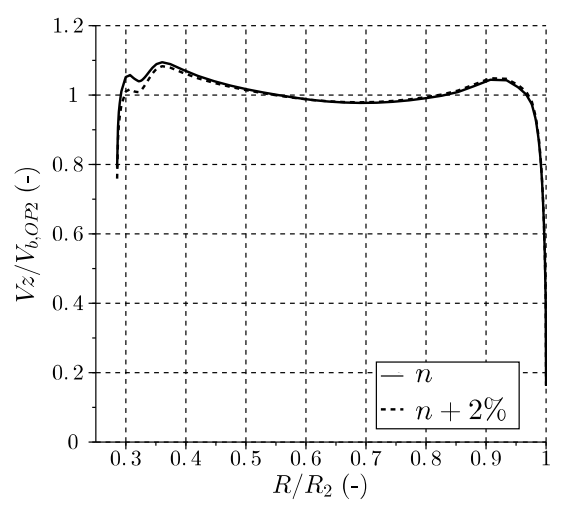

(a) Axial velocity

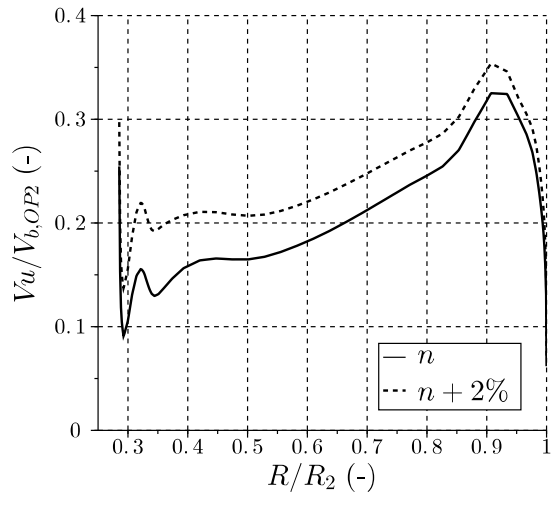

(b) Tangential velocity

Fig. 5 Velocity profiles at the inlet for OP2 with runner rotation speed $n$ and $n+2 \%$

the draft tube inlet when the flow rate is unchanged. Velocity profiles obtained at the three stations in the draft tube with these new inlet boundary conditions are shown in figure 6 . One can observe that not only the level of tangential velocity is now correctly predicted in stations $\mathrm{A}$ and $\mathrm{B}$ but also the prediction of the axial velocity near the center of the draft tube is improved. The low level of axial velocity in this flow region is especially well predicted with LES. It is important to notice that, at station $\mathrm{C}$, the prediction of the velocity profiles is improved especially for the axial velocity close to the axis. However, discrepancies with the experimental measurements still remain and further investigations need to be performed to reach a better agreement.

\subsection{Validation of the simulations on a propeller curve}

Numerical simulations for the off-design points are also validated by comparison with LDV velocity profiles in the draft tube. The runner rotation speed in the RANS calculations of the guide vane - runner used as inlet conditions is here unchanged and equal to the real rotation speed $n$ of the turbine.

The numerical and experimental velocity profiles for OP1 are compared in figure 7. Under part load conditions, a helical vortex rope is formed due to vortex breakdown in the draft tube [29]. This is characterized by a large central region of low axial velocity associated with an increase of tangential velocity from the center of the draft tube. This region increases in size along the draft tube so that the vortex rope takes up the whole section in the diffuser of the draft tube (station C) [29]. Under part load conditions, the inlet tangential velocity of the draft tube is higher than at the best efficiency point according to the velocity triangle at the runner outlet [30]. The tangential velocity is thus higher at OP1 than at OP2 on stations A and B. The same conclusions as for OP2 can be drawn for OP1 but without changing the runner rotation speed for OP1. Similarly to OP2, an improved velocity profiles prediction is obtained with LES compared to URANS. 


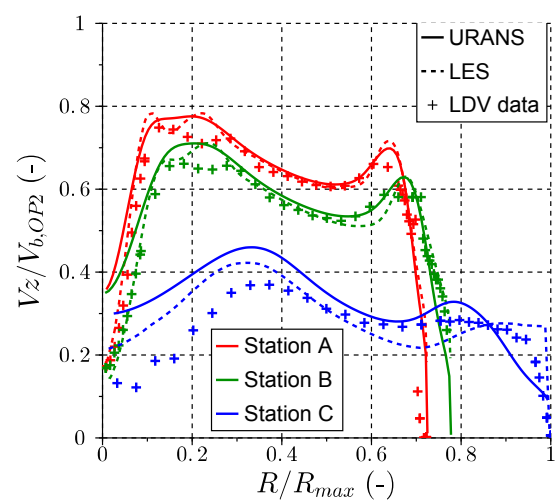

(a) Axial velocity

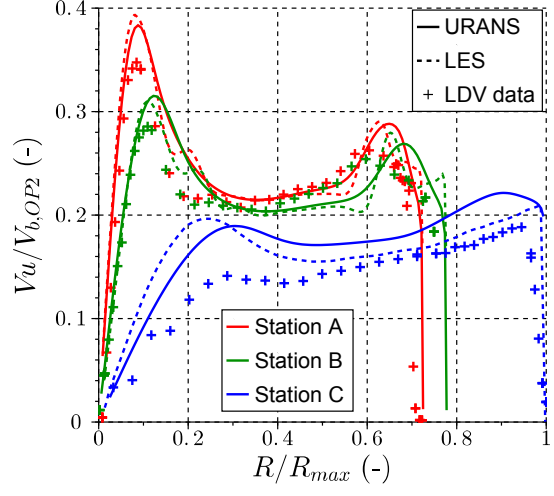

(b) Tangential velocity

Fig. 6 Non-dimensional axial (a) and tangential (b) velocity profiles ( $\mathrm{Vz}, \mathrm{Vu}$ ) versus the non-dimensional radial position for OP2 with inlet velocity profiles corresponding to runner speed $n+2 \%$

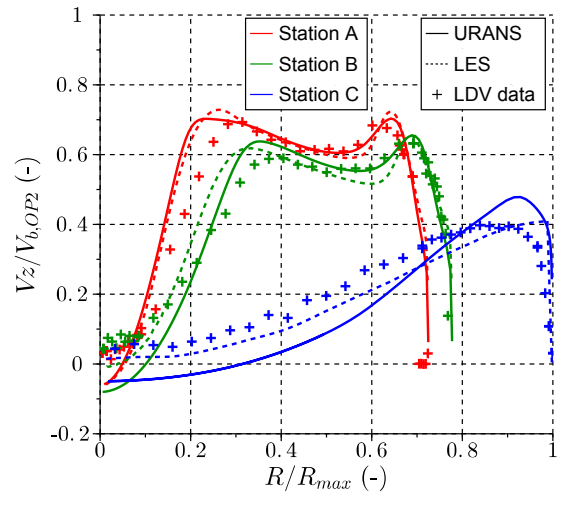

(a) Axial velocity

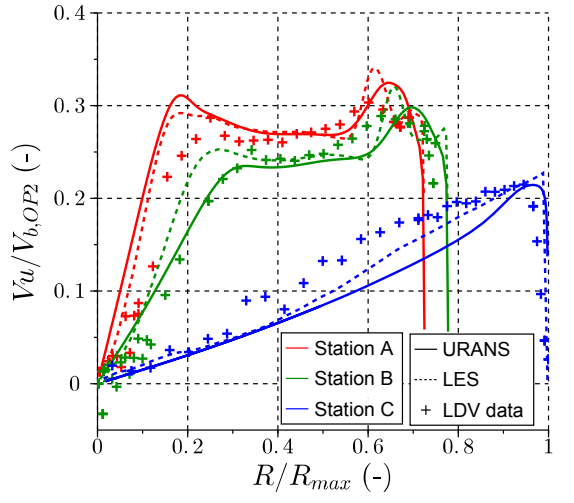

(b) Tangential velocity

Fig. 7 Non-dimensional axial (a) and tangential (b) velocity profiles (Vz, Vu) versus the non-dimensional radial position for OP1

The velocity profiles for OP3 are shown in figure 8 . The high load vortex rope is characterized in stations $\mathrm{A}$ and $\mathrm{B}$ by an increase of the axial velocity from the near wall zone to the center of the draft tube associated with an inversion of the sign of the tangential velocity, which reaches a peak next to the center of the draft tube [29]. One may notice that, similarly to the OP2 case, a systematic error exists between numerical and experimental tangential velocity profiles in stations A and B. Thus the inlet tangential velocity is ill-predicted similarly to the OP2 case, however the downstream consequences are less dramatic. This is attributable to the fact that this off-design point presents large instabilities and particularly a well-established 
vortex rope. The downstream flow predictions are consequently less dependent on variations of the inlet conditions.

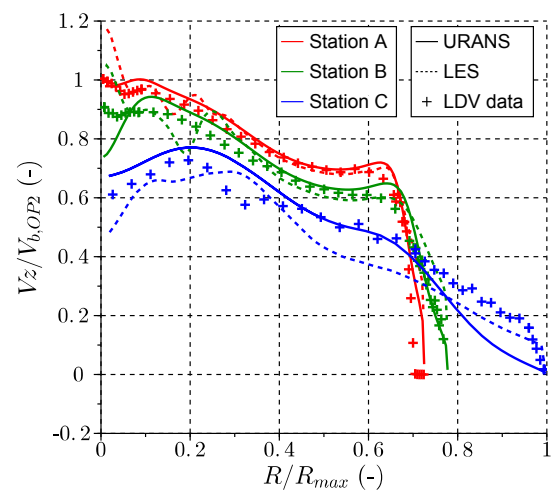

(a) Axial velocity

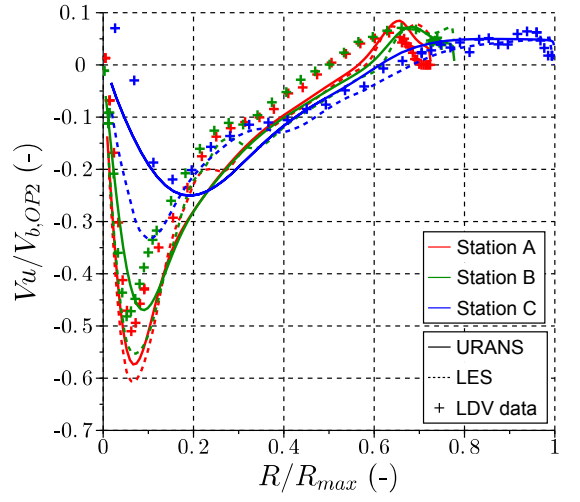

(b) Tangential velocity

Fig. 8 Non-dimensional axial (a) and tangential (b) velocity profiles (Vz, Vu) versus the non-dimensional radial position for OP3

\subsection{Flow topology}

We now concentrate on the flow topology in the draft tube for the three operating points. Coherent vortices are identified using the Q criterion [31]. Vortical structures in the draft tube, predicted by LES and URANS simulations, are presented in figure 9 for the three operating points. The iso-surfaces of positive Q criterion are colored by the axial vorticity (along $z$ axis). Note that the vorticity corresponding to turbine rotation is negative. One can first observe that more vortex structures are resolved with LES than with URANS simulations, in particular parietal turbulent structures are observed in LES. For all operating points, two main regions of vortical structures are observed. The first one is located next to the outer wall and the runner tip and has a helical structure. This corresponds to the propagation of the blade and hub vortices from the runner into the draft tube. The second vortical structure is a central structure generated on the runner tip. Its shape is different for the three operating points. At OP1, both approaches predict a central vortex structure which is rotating in the same direction of rotation as the runner (negative axial vorticity). URANS simulation predicts a smooth diverging central vortex structure whereas with LES, this structure has a helical shape. This is characteristic of the partial load vortex rope [29]. Note that, the classical industrial approach used in this work, based on URANS with the $k-\omega$ SST model, fails to predict the helical shape of the vortex rope for this regime. As previously pointed out, more sophisticated statistical turbulence models may allow to reproduce more details of the vortex rope topology.

A central vortex structure which is rotating in the same direction as the runner is also predicted at the optimal operating point, OP2. For the high load case, OP3, the 
central vortex structure is counter-rotating according to the runner rotation (positive axial vorticity). This is characteristic of the high load vortex rope [29].

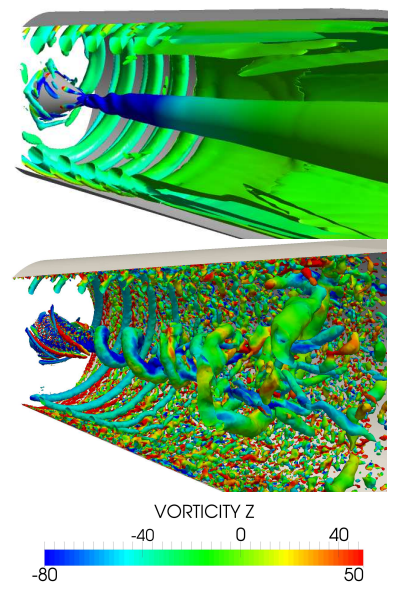

(a) OP1

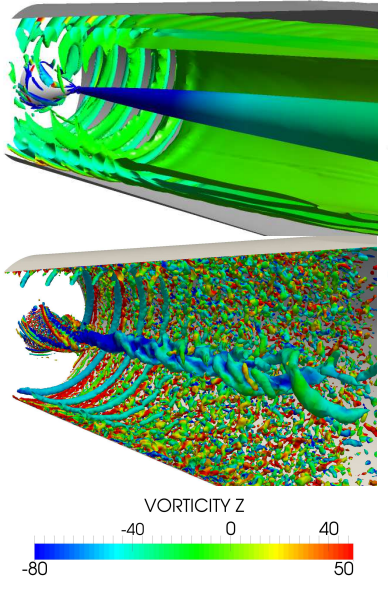

(b) OP2

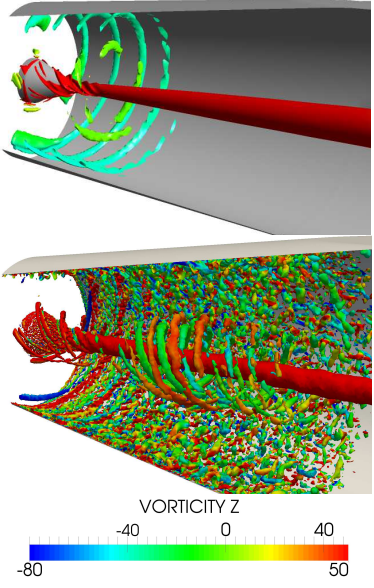

(c) OP3

Fig. 9 Coherent vortices in the draft tube at the three operating points (shown by iso-surface of $\mathrm{Q}$ criterion colored by the vorticity along the $z$ axis (see figure $1 \mathrm{~b}$ )); top: URANS results; bottom: LES results

\section{Physical analysis of head losses in the draft tube}

As explained in the introduction, one of the most important engineering quantity of interest in the draft tube is the head loss since it characterizes the draft tube performance. Thanks to numerical simulations, not only the integral value of the head loss can be obtained but also a better understanding of detailed flow phenomena responsible for it. This section is devoted to the physical analysis of the head losses in the draft tube using URANS and LES results.

\subsection{Head losses equation}

The head $H$ is defined as the total energy, sum of kinetic, static pressure and potential energies, per unit mass of fluid at a given point in the flow, expressed in meters [32]:

$$
H=\frac{p}{\rho g}+z+\frac{V^{2}}{2 g}
$$

where $\rho$ is the density of the fluid, $g$ is the acceleration due to gravity, $z$ is the local elevation and $V$ and $p$ are respectively the velocity and static pressure. As explained in the introduction, the purpose of the draft tube is to convert the residual kinetic 
energy at the runner outlet into static pressure. Hence, the head losses in the draft tube correspond to the total energy loss due to the kinetic energy which is not converted into static pressure but into heat through viscous dissipation.

The flow in the draft tube is governed by the Navier-Stokes equations for an incompressible turbulent flow. The equation for the mean kinetic energy can be derived from Navier-Stokes equations and leads to the following equation (note that the operator $\langle a\rangle$ corresponds to the time averaged value of $a$ obtained from flow statistics):

$$
\begin{array}{r}
\frac{\partial}{\partial x_{j}}\left(\frac{\langle p\rangle}{\rho g}+z+\frac{1}{2 g}\left\langle u_{i}\right\rangle\left\langle u_{i}\right\rangle\right) g\left\langle u_{j}\right\rangle \\
=\underbrace{2 \nu \frac{\partial}{\partial x_{j}}\left(\left\langle u_{i}\right\rangle\left\langle S_{i j}\right\rangle\right)}_{I}-\underbrace{\frac{\partial}{\partial x_{j}}\left(\left\langle u_{i}\right\rangle\left\langle u_{i}^{\prime} u_{j}^{\prime}\right\rangle\right)}_{I I}-\underbrace{2 \nu\left\langle S_{i j}\right\rangle\left\langle S_{i j}\right\rangle}_{I I I}+\underbrace{\left\langle u_{i}^{\prime} u_{j}^{\prime}\right\rangle\left\langle S_{i j}\right\rangle}_{I V}
\end{array}
$$

where $\nu$ is the fluid kinematic viscosity, $u_{i}$ is the velocity in $x_{i}$ direction with $u_{i}=\left\langle u_{i}\right\rangle+u_{i}^{\prime}$, and $S_{i j}=\frac{1}{2}\left(\frac{\partial u_{i}}{\partial x_{j}}+\frac{\partial u_{j}}{\partial x_{i}}\right)$ is strain rate tensor. Terms $(I)$ and $(I I)$ correspond to diffusion of mean kinetic energy. Term $(I I I)$ corresponds to the viscous dissipation of mean kinetic energy. Term $(I V)$ is responsible for the transfer of mean kinetic energy to the turbulent kinetic energy i.e. for turbulent kinetic energy production. This turbulent kinetic energy is then dissipated by the small scales of turbulence through viscous dissipation [33].

Since the total head loss in the draft tube corresponds to an integral value, equation (2) has to be integrated over the total volume $V$ of the draft tube. Lets designate by $S$ its external surface and by $n_{j}$ the $j^{\text {th }}$ component of the outer-pointing normal. By using the divergence theorem, the energy balance in the draft tube becomes :

$$
\begin{array}{r}
\iint_{S}\left(\frac{\langle p\rangle}{\rho g}+z+\frac{1}{2 g}\left\langle u_{i}\right\rangle\left\langle u_{i}\right\rangle\right) g\left\langle u_{j}\right\rangle n_{j} d S \\
=2 \nu \iint_{S}\left(\left\langle u_{i}\right\rangle\left\langle S_{i j}\right\rangle\right) n_{j} d S-\iint_{S}\left(\left\langle u_{i}\right\rangle\left\langle u_{i}^{\prime} u_{j}^{\prime}\right\rangle\right) n_{j} d S \\
-2 \nu \iiint_{V}\left\langle S_{i j}\right\rangle\left\langle S_{i j}\right\rangle d V+\iiint_{V}\left\langle u_{i}^{\prime} u_{j}^{\prime}\right\rangle\left\langle S_{i j}\right\rangle d V
\end{array}
$$

On the left-hand side of equation (3), one can recognize the head flux through the draft tube external surface. This flux is obviously zero through the lateral solid surfaces which are impermeable. The assumption of a steady flow with constant flow rate $Q$ and uniform head $H$ on the inlet (suffix $i n$ ) and outlet (suffix out) surfaces of the draft tube then leads to:

$$
\begin{array}{r}
\iint_{S_{\text {in }}}\left(\frac{\langle p\rangle}{\rho g}+z+\frac{1}{2 g}\left\langle u_{i}\right\rangle\left\langle u_{i}\right\rangle\right) g\left\langle u_{z}\right\rangle d S-\iint_{S_{\text {out }}}\left(\frac{\langle p\rangle}{\rho g}+z+\frac{1}{2 g}\left\langle u_{i}\right\rangle\left\langle u_{i}\right\rangle\right) g\left\langle u_{z}\right\rangle d S \\
=g Q\left(H_{\text {in }}-H_{\text {out }}\right)
\end{array}
$$

One thus obtains the expression for the total head loss $\Delta H=H_{\text {in }}-H_{\text {out }}$. 
Physical phenomena responsible for head losses

According to equations (3) and (4), the head losses in the draft tube are influenced by four terms :

$$
\begin{aligned}
g Q \Delta H=- & \underbrace{2 \nu \iint_{S}\left(\left\langle u_{i}\right\rangle\left\langle S_{i j}\right\rangle\right) n_{j} d S}_{I}+\underbrace{\iint_{S}\left(\left\langle u_{i}\right\rangle\left\langle u_{i}^{\prime} u_{j}^{\prime}\right\rangle\right) n_{j} d S}_{I I} \\
& +\underbrace{2 \nu \iiint_{V}\left\langle S_{i j}\right\rangle\left\langle S_{i j}\right\rangle d V}_{I I I}+\underbrace{\iiint_{V}-\left\langle u_{i}^{\prime} u_{j}^{\prime}\right\rangle\left\langle S_{i j}\right\rangle d V}_{I V}
\end{aligned}
$$

On the right-hand side of equation (5), terms $(I)$ and $(I I)$ correspond to the diffusion of mean kinetic energy. Terms $(I I I)$ and $(I V)$ contribute to head losses by viscous dissipation of the mean kinetic energy either directly by the mean flow or indirectly after turbulent kinetic energy production.

4.2 Turbulence modelling and head losses prediction

In LES and URANS simulations, only filtered and mean values (according to the Reynolds decomposition) of pressure and velocity are respectively resolved and turbulence modelling is used to take into account the effect of the unresolved part of the flow on the resolved one. Lets designate by $\bar{a}$ the mean value of $a$ in case of a URANS calculation and the filtered value of $a$ in the LES case. Equation (5) then yields:

$$
\begin{array}{r}
\iint_{S_{i n}}\left(\frac{\langle\overline{P *}\rangle}{\rho g}+\frac{1}{2 g}\left\langle\overline{u_{i}}\right\rangle\left\langle\overline{u_{i}}\right\rangle\right) g\left\langle\overline{u_{z}}\right\rangle d S-\iint_{S_{\text {out }}}\left(\frac{\langle\overline{P *}\rangle}{\rho g}+\frac{1}{2 g}\left\langle\overline{u_{i}}\right\rangle\left\langle\overline{u_{i}}\right\rangle\right) g\left\langle\overline{u_{z}}\right\rangle d S \\
=-\underbrace{\iint_{S}\left(2\left\langle\overline{u_{i}}\right\rangle\left(\nu\left\langle\overline{S_{i j}}\right\rangle+\left\langle\nu_{t} \overline{S_{i j}}\right\rangle\right) n_{j}\right) d S}_{I I I}+\int \underbrace{\iint_{S}\left\langle\overline{u_{i}}\right\rangle\left\langle{\overline{u_{i}}}^{\prime} \overline{u_{j}}{ }^{\prime}\right\rangle n_{j} d S}_{I V} \\
+\underbrace{\iiint_{V} 2 \nu\left\langle\overline{S_{i j}}\right\rangle\left\langle\overline{S_{i j}}\right\rangle d V}_{I V}+\underbrace{\iiint_{V}-\left\langle\bar{u}_{i}^{\prime} \overline{u_{j}}\right\rangle\left\langle\overline{S_{i j}}\right\rangle d V}_{V}+\underbrace{\iiint_{V} 2\left\langle\nu_{t} \overline{S_{i j}}\right\rangle\left\langle\overline{S_{i j}}\right\rangle d V}_{V}
\end{array}
$$

where $\langle\overline{P *}\rangle$ is the modified pressure. $\nu_{t}$ corresponds to the turbulent viscosity in the case of a URANS calculation or to the eddy viscosity in the case of LES. We concentrate on the different terms of the right-hand side of equation (6). The terms involving the viscosity $\nu_{t}$ are dependent on the model used for the simulation: $k-\omega$ SST model for the URANS calculations and the dynamic Smagorinsky model for the LES. Terms $(I)$ and $(I I)$ correspond to the diffusion of mean kinetic energy and term $(I I I)$ is the viscous dissipation of mean kinetic energy. The turbulent kinetic 
energy production decomposes into a $(I V)$ resolved and a $(V)$ modelled part which involves the turbulent viscosity $\nu_{t}$.

Two levels of analysis of head losses are performed in this work. An overall analysis is first conducted which consists of calculating the different terms of equation (6) in order to determine the leading terms responsible for the head losses. A local analysis is then performed to investigate the spatial distribution of these leading terms within the draft tube. Similar analysis has been performed experimentally in [34] and numerically in [35] in order to study energy losses in a turbine cascade. It was concluded that the viscous dissipation of turbulent kinetic energy produced from the mean flow predominates in total pressure losses generation.

\section{Best efficiency point $O P^{2}$}

The terms of equation (6) have been calculated both for LES and URANS computations for all operating points. The conclusions are similar for the three operating points and are only shown for the best efficiency point, OP2. It is important to check the ability for the numerics to accurately reproduce the balance between the left hand side and the right hand side of equation (6). We have observed that the URANS leads to a difference of $4.8 \%$ when it is only of $0.02 \%$ for the LES.

Surface integrals in equation (6) are found to be negligible in front of volume integrals in the energy balance. We note $P_{M}=\iiint_{V} 2\left\langle\nu_{t} \overline{S_{i j}}\right\rangle\left\langle\overline{S_{i j}}\right\rangle d V$ and $P_{R}=$ $\iiint_{V}-\left\langle\overline{u_{i}} \bar{u}^{\prime}\right\rangle\left\langle\overline{S_{i j}}\right\rangle d V$ the two parts of the turbulent kinetic energy production which are respectively modelled and resolved. $D=\iiint_{V} 2 \nu\left\langle\overline{S_{i j}}\right\rangle\left\langle\overline{S_{i j}}\right\rangle d V$ is the viscous dissipation of the mean kinetic energy. Note that the viscous dissipation by the small scales of turbulence does not appear explicitly but implicitly through $P_{M}$.

The contribution of $P_{R}, P_{M}$ and $D$ to the head losses $(g Q \Delta H)$ are compared for the URANS simulation and LES of OP2 in figure 10. We may remark that the part of the viscous dissipation $D$ in the head losses is negligible for both simulations. The production of turbulent kinetic energy, represented by $P_{M}$ and $P_{R}$, constitutes the main contribution to the head losses in the draft tube. In URANS simulations, the turbulent flow is almost totally modelled, explaining the large value of $P_{M}$. Conversely, the resolved turbulent kinetic energy production $P_{R}$ is negligible in the URANS simulation. In the LES, a large part of the turbulent fluctuations is resolved leading to a dominant contribution of $P_{R}$. However, the subgrid part of the turbulent flow is modelled so that $P_{M}$ is not zero. As expected, the head losses are thus more dependent on the turbulence model in the URANS simulation than in the LES. Moreover, in LES, the modelled part of turbulent kinetic energy production can be reduced by refining the mesh since it is associated with the contribution of the subgrid scales.

Local analysis of head losses in the draft tube for OP2

Head losses are thus strongly correlated with the turbulent kinetic energy production, which is principally modelled in URANS and partially resolved in LES. In order to identify the hydrodynamic phenomena responsible for turbulent production and thereby for head losses in the draft tube, the distributions of $P_{M}$ for the URANS simulation and of $P_{M}$ and $P_{R}$ for the LES are shown in figure 11 for OP2. In LES, $P_{R}$ dominates but $P_{M}$ remains responsible for a part of the head losses, principally in the vortex rope. This is confirmed by the $P_{M}$ and $P_{R}$ profiles in figure 12 obtained 


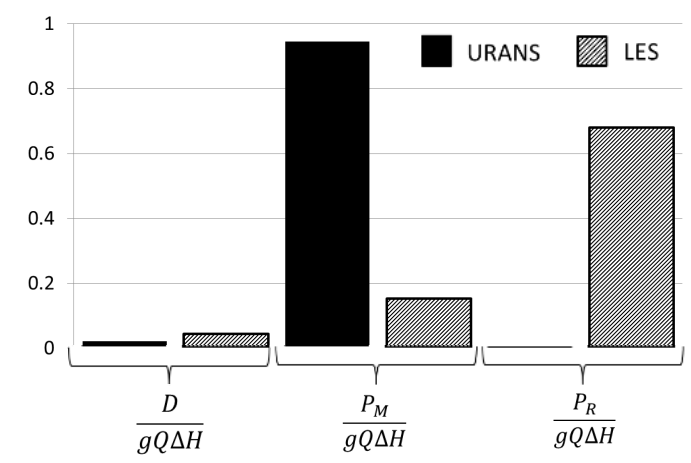

Fig. 10 Contribution of $P_{R}, P_{M}$ and $D$ to the head loss $(g Q \Delta H)$ for OP2 in URANS and LES results

through azimuthal averaging on a plane which location is defined by the dotted lines in figure 11. Maximum values of $P_{M}$ and $P_{R}$ are observed next to the draft tube center $\left(R / R_{\max }=0.1\right)$ and next to the wall $\left(R / R_{\max }\right.$ close to 1$)$.

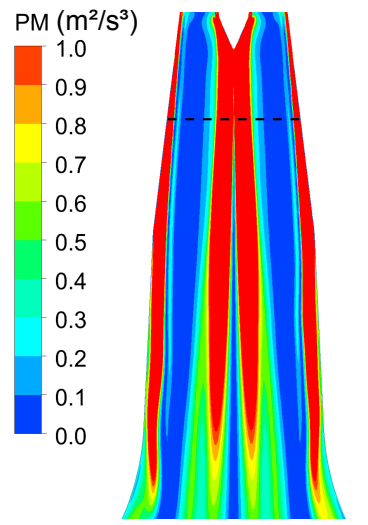

(a) URANS
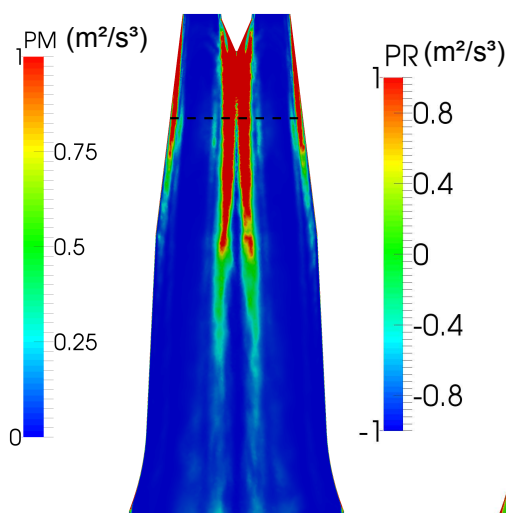

(b) LES

Fig. 11 Distribution of $P_{M}$ and $P_{R}$ predicted by the LES and URANS simulation for OP2 on a median plane in the draft tube

This is supported by the comparison of the Reynolds stresses measured at the different stations and obtained by LES in the figure 13. Only the three components $\left\langle V_{u}^{\prime} V_{u}^{\prime}\right\rangle,\left\langle V_{z}^{\prime} V_{z}^{\prime}\right\rangle$ and $\left\langle V_{u}^{\prime} V_{z}^{\prime}\right\rangle$ of the Reynolds stress can be evaluated experimentally since the radial velocity $V_{r}$ is not measured by LDV. Moreover, these Reynolds stresses can be calculated from LES results, but not from URANS results where the Reynolds stress is modelled. It is important to notice that the shape of the Reynolds stresses profiles is well predicted by LES with peak values near the wall 


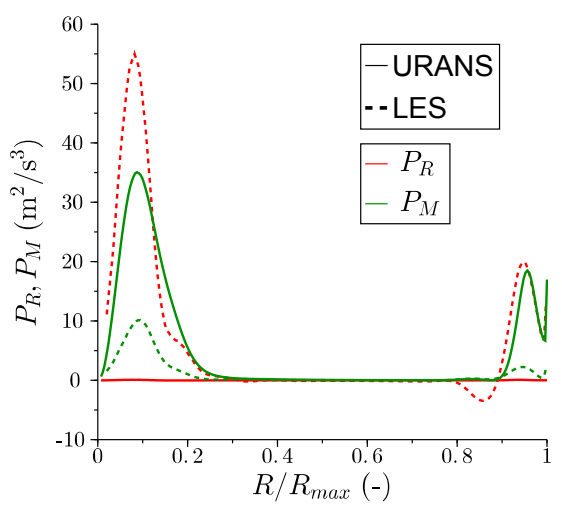

Fig. 12 Profiles of $P_{M}$ and $P_{R}$ predicted by the LES and URANS simulation for OP2 on a plane perpendicular to $z$ axis in the draft tube

and in the center of the draft tube. Moreover, these high values of Reynolds stresses are associated to high velocity gradients, as shown in figure 6, thus leading to turbulent production observed in figure 11. However this Reynolds stress comparison necessitates a more precise evaluation of the origin of the experimental uncertainties in particular at section $\mathrm{C}$ which presents a very large dispersion near the outer wall.

In LES, $P_{R}$ reaches higher values than $P_{M}$ in agreement with the integral values in figure 10. The flow regions where $P_{M}$ is found to be non-negligible in LES are those where the mesh should be refined in order to reduce the influence of the turbulence model on the prediction. Negative values of $P_{R}$ in LES correspond to inverse energy transfer (backscatter) that is to say to energy transfer from the turbulent flow to the mean flow [36].

According to the distributions of $P_{M}$ and $P_{R}$ observed in URANS and LES, head losses occur in the center of the draft tube, because of the presence of the central vortex structures observed in figure 9 . Head losses are also found to be important in the vicinity of the external wall, only in the cone for the LES and in the whole draft tube for the URANS simulation. These head losses next to the diffuser wall for URANS are attributable to axial velocity gradients prediction which is higher than experimentally observed and than in the LES for OP2 (see station C in figure 6).

We next calculate the right-hand side of equation (6) over a control volume $V$ located between the inlet plane $(z=0)$ and the $z$ plane. Moreover, $V$ is decomposed into two volumes: one covering the central region of the draft tube, where head losses occur due to the vortex rope, the other one covering head losses next to the wall. The head losses evolution as a function of $z$ is represented for URANS and LES for OP2 point in figure 14. Note that figure 14 corresponds to the cumulated value of the head losses between the origin $z=0$ and a given $z$ location. Head losses are found to be higher in the near wall zone than in the center of the draft tube. A strong increase of these near wall losses is observed from the inlet of the draft tube until the end of the cone $\left(z / z_{\max }=0.45\right)$. Indeed, as shown in figure 12 , the turbulent production profiles $P_{M}$ and $P_{R}$ are smaller next to the outer wall than near the draft tube axis but they are acting on a much larger volume than the one of the vortex 


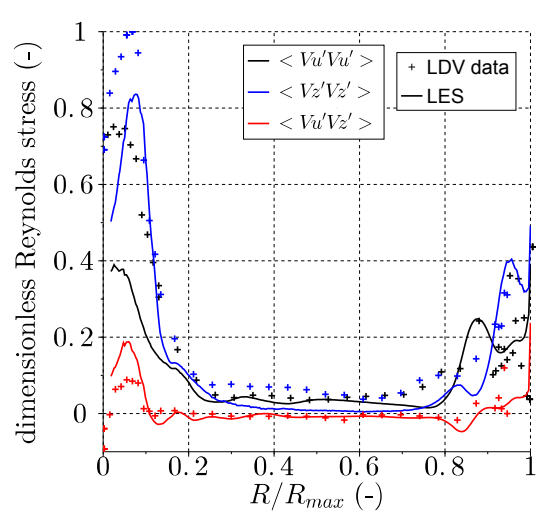

(a) Station A

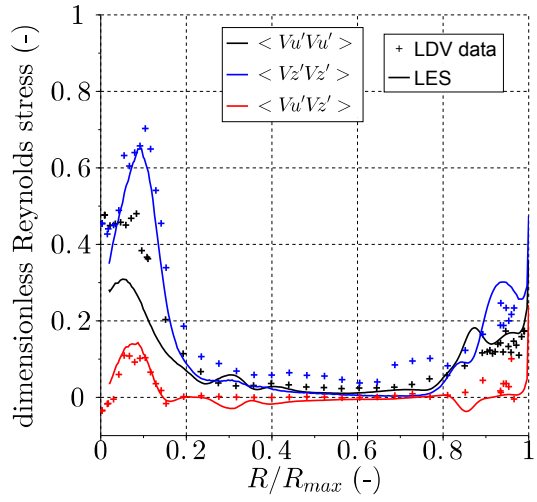

(b) Station B

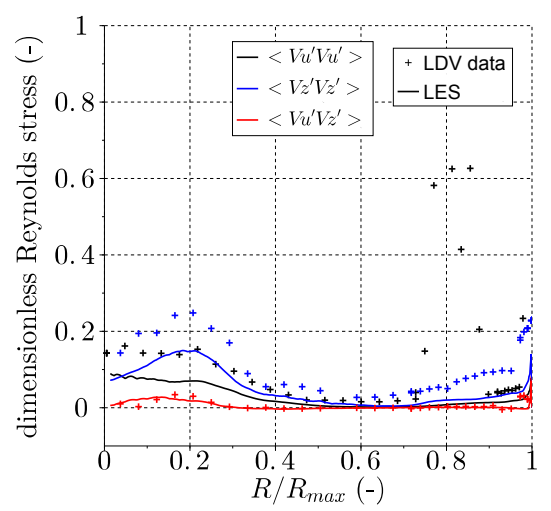

(c) Station C

Fig. 13 Reynolds stresses profiles at the three stations in the draft tube for OP2 obtained from experimental measurements (symbols) and LES (lines) ; $R_{\max }$ corresponds to the maximum radius value at each station

rope concentrated near the axis. Head losses in the near wall region are similar in URANS and LES until the end of the cone $\left(z / z_{\max }=0.45\right)$ where head losses keep increasing in URANS whereas they level off in LES. This has been observed in figure 11 and is attributable to the higher velocity gradients next to the diffuser wall in URANS. Conversely, head losses in the central zone are slightly higher in LES than in URANS. Indeed, higher axial velocity gradients are predicted with LES than with URANS in this flow region (see figure 6), in agreement with experimental velocity profiles. However, the increase of head losses in the central region is slower than in the near wall region. As a consequence, the global head losses are higher in URANS than in LES. This is an indication of the more dissipative nature of the URANS computation as compared to the LES simulation as previously shown by $[37,38]$. It is important to remark that the downstream evolution of the various components of the head losses shown on figure 14 is difficult to measure experimentally and 
renders any precise comparison with experimental data impossible at the present time. However, the better prediction of the downstream evolution of the velocity profiles by the LES may be a sign of a better head losses prediction. Figure 14 furthermore shows that head losses mechanisms are quite different depending on the flow region: just downstream of the turbine, in the cone or further downstream in the draft tube. This demonstrates that considering only the global head losses between the inlet and the outlet of the tube does not constitute a proper test for a turbulent simulation. A proper model must indeed be able to properly reproduce the losses in the different flow regions in order to be predictive for various draft tube geometries.

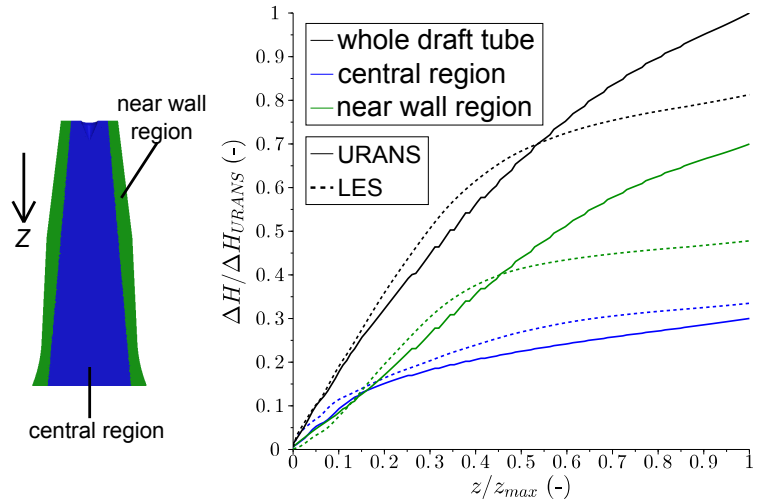

Fig. 14 Evolution of the head losses (non-dimensional) along the draft tube predicted by the URANS calculation and LES for OP2 and decomposition of the head losses in the central and near wall regions of the draft tube

\subsection{Physical analysis of head losses along a propeller curve}

Physical and hydrodynamic phenomena responsible for head losses in the draft tube have been identify for the best efficiency point OP2. The same analysis can be conducted on OP1 and OP3 in order to understand the head losses variation with the flow rate. Since the influence of the turbulence modelling on head losses prediction is lower in LES than in URANS, analysis is shown here using LES results.

The global head losses as well as the evolutions of the non-dimensional head losses along the draft tube are shown in figure 15 for the three considered operating points. Note that figure 15b corresponds to the cumulated value of the head losses between the origin $z=0$ and a given $z$ location. The points shown on figure 15a therefore correspond to the values obtained for $z / z_{\max }=1$ in figure $15 \mathrm{~b}$. The head losses are the lowest for OP2, which is the best efficiency point of the turbine, and the highest for OP1, which corresponds to low flow rate. For OP2, head losses are increasing from the inlet to approximately the half length of the draft tube where they then level off. For the off-design points, OP1 and OP3, head losses are increasing along the whole draft tube but the highest increase is also found in the first part of the draft tube, for $z / z_{\max }$ smaller than 0.5 , in particular for OP1. 


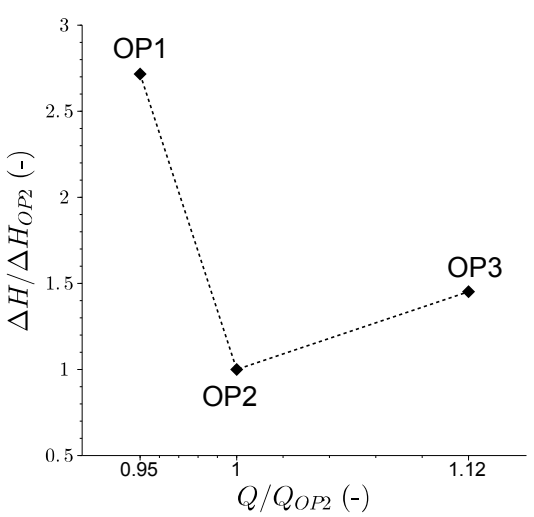

(a)

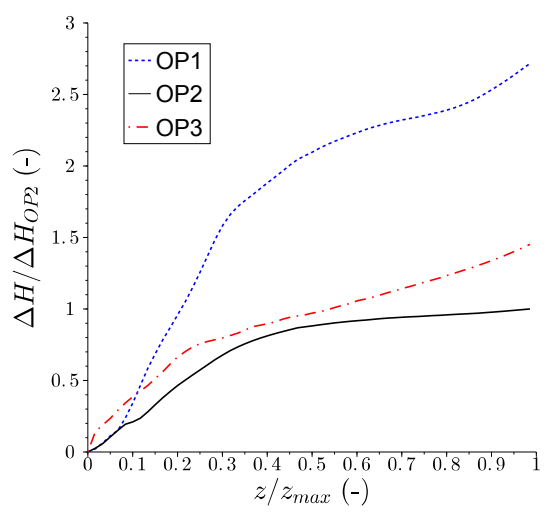

(b)

Fig. 15 Evolutions of the non-dimensional head losses in the draft tube for the three operating points; (a) integral values of head losses and (b) evolution of head losses along the draft tube

In order to better understand the different evolutions of head losses with the flow rate, the distributions of the dominating term in equation (6), $P_{R}$, are shown in figure 16 on a median plane in the draft tube for OP1, OP2 and OP3. According to the distributions of $P_{R}$ for all operating points, head losses are occurring in the center of the draft tube due to the central vortex structures observed in figure 9 and in the near wall region. In particular for OP1, the diverging vortex rope observed in figure 9a seems to be responsible for a large part for the losses. Turbulent production $P_{R}$ is principally concentrated in the cone of the draft tube for OP2 whereas it occurs along the whole draft tube for OP1 and OP3. This is in agreement with the head losses evolution in figure $15 \mathrm{~b}$.

Notice that the averaged isocurves remain quite irregular especially for the OP1 and OP3 operating points. This is due to the highly intermittent turbulent flow for these two cases. The achievement of smoother curves would require a much longer time integration but the main conclusions drawn from figure 16 would be identical.

For further analysis, the head losses evolutions are decomposed in figure 17 into head losses in the central region and in the vicinity of the wall, as explained in section 4.2. These regions are indicated in figure 16 for clarity. Similarly to figure $15 \mathrm{~b}$, figure $17 \mathrm{~b}$ represents the cumulative head losses between the inlet $z=0$ and a given $z$ location. The points shown on figure 17a therefore correspond to the values obtained for $z / z_{\max }=1$ in figure $17 \mathrm{~b}$.

From figure $17 \mathrm{a}$, it is clear that the increase of head losses from OP2 to OP1 is due to the central vortex structure which leads to higher turbulent production than at OP2. By contrast, from OP2 to OP3 head losses increase in both the central region and the near wall region. Similar evolutions of head losses in the central and near wall regions are found for OP2 and OP3: head losses strongly increase in the first part of the draft tube and then level off. Moreover, head losses are higher in the near wall region than in the center of the draft tube for these two points. This is the contrary for OP1 where head losses in the near wall region are lower than in 


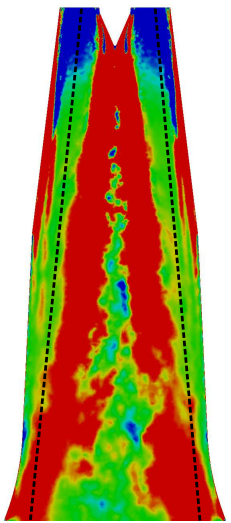

(a) OP1

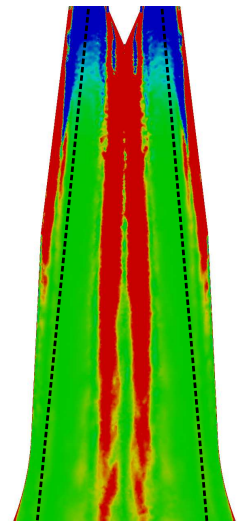

(b) OP2

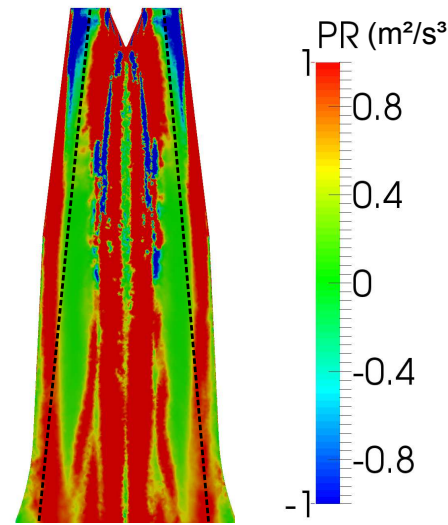

(c) OP3

Fig. 16 Distribution of $P_{R}$ predicted by the LES for the three operating points on a median plane in the draft tube

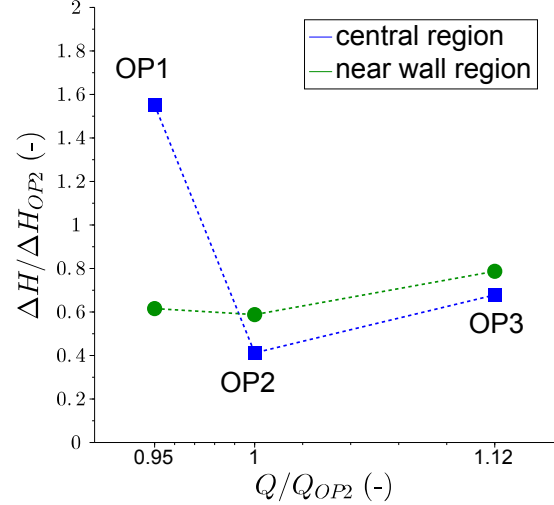

(a)

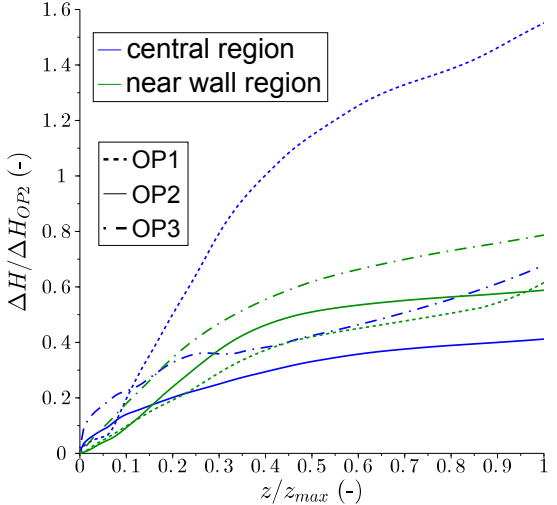

(b)

Fig. 17 Evolutions of the non-dimensional head losses in the central and near wall region in the draft tube for the three operating points; (a) integral values of head losses and (b) evolution of head losses along the draft tube

the central part. These are the lowest of the near wall region losses among the three operating points along almost the whole draft tube length. They increase at the end of the draft tube due to the extension of the vortex rope till the near wall region (see figure 16a). Since, the value presented in figure 17 a correspond to the value for $z / z_{\max }=1$ in figure $17 \mathrm{~b}$, head losses in the near wall region for OP1 are slightly higher than those at OP2. Head losses in the central region for OP1 are constantly increasing along the draft tube due to the persistence of the vortex rope. 


\section{Conclusion}

Unsteady RANS (URANS) simulations and Large Eddy Simulations (LES) were performed in the draft tube of a bulb turbine at three different operating points. Inlet boundary conditions of the draft tube accounting for the unsteadiness from the runner have been prescribed. The main objectives of the present study were (i) to evaluate the reliability of the numerical approaches and (ii) to better understand the origin of the head losses in the draft tube.

Predicted velocity profiles in the draft tube are compared with experimental measurements in order to assess the validity of the numerical simulations. Accurate tangential velocity profile at the draft tube inlet is found to be crucial for a correct flow prediction in the draft tube. For the best efficiency point of the turbine, a slight increase of the inlet tangential velocity level exported from the guide vane - runner calculation is thus necessary to obtain more realistic flow prediction in the draft tube. In order to properly quantify the flow sensitivity to inlet conditions, uncertainties quantification on the flow prediction in the draft tube will be investigated in further work. For the off-design points, the velocity profiles prediction is better in the cone due to a correct inlet tangential velocity level predicted by the guide vane - runner calculation. Flow prediction has however to be improved in the diffuser of the draft tube for the three operating points. A detailed investigation of the energy dissipation in this flow region shows that a large proportion of energy loss takes place in the vicinity of the diffuser wall. We therefore plan to perform further LES studies with mesh refinement next to the diffuser wall. URANS and LES simulations globally predict similar large scale flow structures in the draft tube. However, LES are able to reproduce the complexity of the vortex structures and render possible a better understanding of the vortex dynamics.

Using the mean kinetic energy balance in the draft tube, the physical and hydrodynamic phenomena responsible for head losses are identified. As expected, head losses are mainly due to turbulent kinetic energy dissipation and thus are strongly correlated with the turbulent kinetic energy production from the mean kinetic energy. Head losses prediction in URANS simulations is highly dependent on the turbulence modelling. On the other hand, the head losses are mainly controlled by the resolved structures in LES and only a small part of the head losses is dependent on the subgrid scale modelled flow. The modelled part of the head losses in LES acts mainly in the vortex rope and can be reduced by refining the mesh. This suggests that an improvement of the head losses prediction can be expected from LES on refined mesh. This constitutes an advantage of LES, because improvement of URANS prediction cannot be expected without a complex specific tuning of the model.

Thanks to a more local analysis of the energy balance in the draft tube, the central vortex structure is found to be responsible for head losses, as well as velocity gradients in the vicinity of the external wall. It is shown that the head losses mechanisms are very different just downstream of the turbine, in the cone or further downstream in the draft tube. This constitutes a real challenge for turbulence modelling since the model must be able to properly reproduce these various dissipative mechanisms in order to be predictive for the global draft tube losses. The local energy balance analysis conducted for off-design points highlights the hydrodynamic phenomena at the origin of poor performances of the draft tube under these operating conditions. Under part load conditions, the helical vortex rope is thus responsible for high turbulent production and thus dissipation of mean kinetic energy. Conversely, 
at the best efficiency and high load points, turbulent production in the near wall region is higher than in the vortex rope. The differences in head losses between the best efficiency point and the off-design points are principally due to higher turbulent production in the vortex rope under off-design conditions. The physical analysis of head losses presented in this study enables us to localize the regions of high head losses. This constitutes a valuable information to optimize the draft tube design.

Acknowledgements The authors would like to thank GE Renewable Energy for the financial support and contribution to this project. Vincent Moureau and Ghislain Lartigue from the CORIA lab, and the SUCCESS scientific group are acknowledged for providing the YALES2 code. Computations presented in this paper were performed using HPC resources from GENCIIDRIS (Grant No. 2012-020611) and CIMENT infrastructure (supported by CPER07_13 CIRA and ANR-10-EQPX-29-01).

\section{A Mesh influence study for URANS and LES}

\section{A.1 Mesh convergence study for URANS simulations}

We have performed a classical convergence test consisting in a progressive mesh refinement. Four different meshes have been considered. First, three meshes with an identical averaged $y^{+}$ value equal to 5 : mesh A with 2 million elements; mesh $\mathrm{B}$ with 4 million; mesh $\mathrm{C}$ with 10 million. Second, a fourth mesh (mesh D) with 2 million of elements but a $y^{+}$value smaller than 2 on each wall (mesh D) as recommended by ANSYS CFX to resolve the turbulent boundary layer with the $k-\omega$ model.

In order to more rapidly obtain converged statistics, we use station A to check the mesh convergence. A comparison of the axial and tangential velocity profiles at station A for OP2 are presented in figure 18 . The various meshes yield very close profiles. We have checked that similar results are obtained for the other operating points. The study presented in the manuscript is thus based on URANS simulations performed using the first mesh (mesh A) since it constitutes an "economical" mesh combining sufficient wall resolution and a better resolution of the internal flow as compared with mesh D.

\section{A.2 Mesh influence study for Large Eddy Simulations}

The definition of a correct mesh for LES in complex geometries remains a challenging question. Two major points have indeed to be considered : the near-wall resolution and the discretization of the internal flow.

\section{Near-wall resolution}

First, the near-wall resolution influence has been studied using four meshes with varying $y^{+}$ but with similar mesh size in the internal flow region. The characteristics of the four different meshes are presented in table 2. The first three meshes (mesh1, mesh2 and mesh3) are composed of tetrahedral cells only and the mesh is refined close to the walls. Cells with a too large aspect ratio between the mesh size in the direction parallel to the wall and the one in the normal direction are not allowed ought to numerical convergence criteria. Therefore, even with a very large number of cells of 1400 million (mesh3), the minimum $y^{+}$remains close to 50. We have then used a fourth mesh (mesh4) still consisting of tetrahedral cells away from the wall but with layers of prismatic cells close to the walls. This procedure allows for a better refinement in the near wall region with $y^{+}$values close to 10. Again for numerical convergence reasons, it is not possible to go below this value with the chosen mesh. For the four simulations, we use the wall-function developed by Duprat et al. [39] for flows with strong adverse pressure 


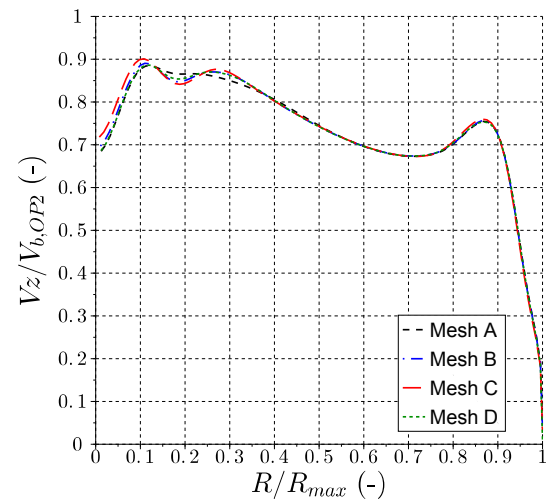

(a) Axial velocity

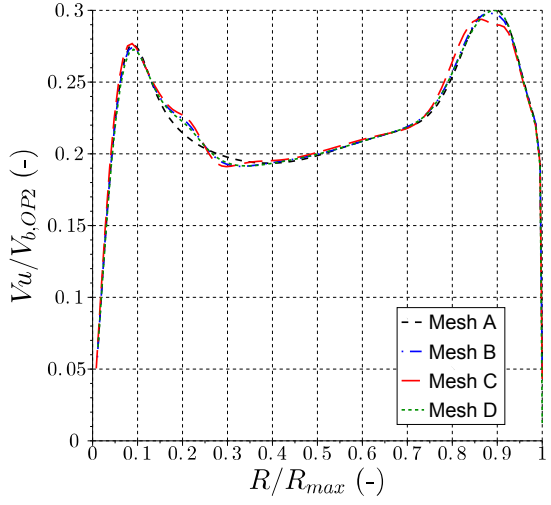

(b) Tangential velocity

Fig. 18 Non-dimensional axial (a) and tangential (b) velocity profiles (Vz, Vu) versus the non-dimensional radial position for OP2 for meshes A to D in URANS

Table 2 Characteristics of the four meshes with the corresponding mean $y^{+}$value

\begin{tabular}{ccccc}
\hline mesh & mesh1 & mesh2 & mesh3 & mesh4 \\
\hline number of elements (in million) & 12 & 28 & 1400 & 16 (with prism layers) \\
\hline number of nodes (in million) & 2.5 & 5.7 & 239 & 4.7 \\
\hline$y^{+}$cone & 400 & 250 & 60 & 12 \\
$y^{+}$diffuser & 750 & 150 & 40 & 8 \\
\hline
\end{tabular}

gradients. A fifth simulation with mesh4 is also performed without wall-function (i.e. applying a no-slip boundary condition at the walls).

Simulations have been performed at the best operating point (OP2). As previously, for time convergence reasons, we consider the statistical quantities in station A to test the mesh convergence. Figure 19 compares the axial and tangential velocity profiles at station A for the simulations with the five different meshes. As compared to the mesh4 case (16 million cells), the very well resolved computation with mesh3 (1400 million cells) does not lead to significant differences. Furthermore, the mesh4 results with or without wall function are very similar. The conclusion of this mesh convergence test is that the relatively economical LES with mesh4 and without any recourse to a wall function are sufficiently resolved to capture the main flow phenomena. The fact that a relatively loose resolution at the wall with $y^{+} \approx 10$ can be utilized can be explained by the presence of the quasi constant adverse pressure gradient in the boundary layer. Indeed, conversely to the classical boundary layer on a flat plane, the adverse pressure gradient boundary layer is less controlled by the wall friction.

\section{discretization of the internal flow}

The discretization quality outside the boundary layers is now addressed. The assessment of the mesh quality is based on the recent work of Benard et al. [40]. This work states that two distinct criteria have to be checked for mesh definition in LES. The first criterion verifies that the mean velocity gradient is sufficiently well described, whereas the second criterion ensures that enough large scale energy is explicitly resolved. In [40], the first criterion is based on the 


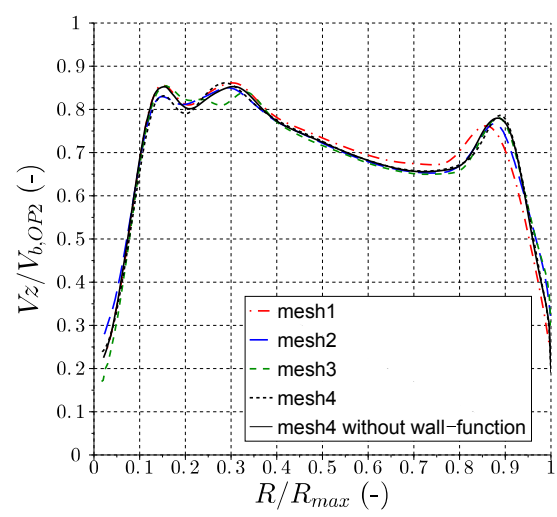

(a) Axial velocity

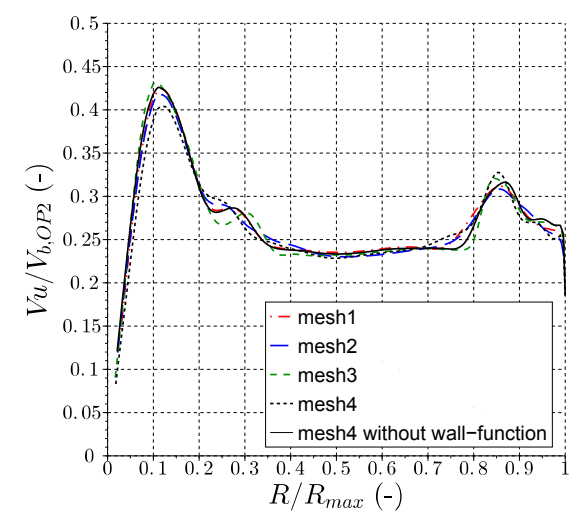

(b) Tangential velocity

Fig. 19 Non-dimensional axial (a) and tangential (b) velocity profiles (Vz, Vu) versus the non-dimensional radial position for OP2 for the meshes 1 to 3 with wall-function and for mesh 4 with and without wall-function

minimization and the homogenization of the quantity $Q c_{1}$, defined as,

$$
Q c_{1}=\Delta^{2} \max _{i, j}\left\{\frac{\partial^{2} u_{i}}{\partial x_{j}^{2}}\right\},
$$

with $\Delta$ the mesh size (see [40] for details). The second criterion is based on Pope's criterion [33], stating that $80 \%$ of the total turbulent kinetic energy has to be explicitly resolved in LES. The second criteria is then based on the quantity $Q c_{2}$,

$$
Q c_{2}=\frac{E_{s g s}}{E_{s g s}+E_{R}}
$$

with $E_{\text {sgs }}$ the subgrid-scale part of the turbulent kinetic energy and $E_{R}$ the resolved part of the turbulent kinetic energy. The mesh is defined to respect $Q c_{2}<0.2$.

The mesh used in this study (mesh4, with 16 million cells) has been defined to keep $Q c_{2}$ smaller than 0.2 on a large majority of cells and to keep $Q c_{1}$ as homogeneous as possible. Starting from this first mesh, the local mesh size is then adapted to reduce the value of $Q c_{1}$ (keeping the same near wall resolution) and to keep $Q c_{2}$ smaller than 0.2 . We have followed this procedure to obtain two new meshes respectively composed of 32 (mesh5) and 120 (mesh6) million cells. Figure 20 shows the probability density functions of $Q c_{1}$ and $Q c_{2}$ for each mesh. Moreover, figure 21 shows that the velocity profiles obtained with these three different meshes are really close.

Finally, the above analysis on near-wall mesh resolution and on discretization of the internal flow demonstrates that mesh4, as described in table 2, allows for a proper flow prediction in the draft tube. The same analysis has been performed for the other operating points which leads to the same conclusion.

\section{References}

1. J. Fonkenell, La Houille Blanche (2), 27 (2003)

2. M.F. Gubin, Draft tubes of hydro-electric stations (Published for the Bureau of Reclamation, US Dept. of the Interior and National Science Foundation, Washington, DC by Amerind Pub. Co., 1973) 


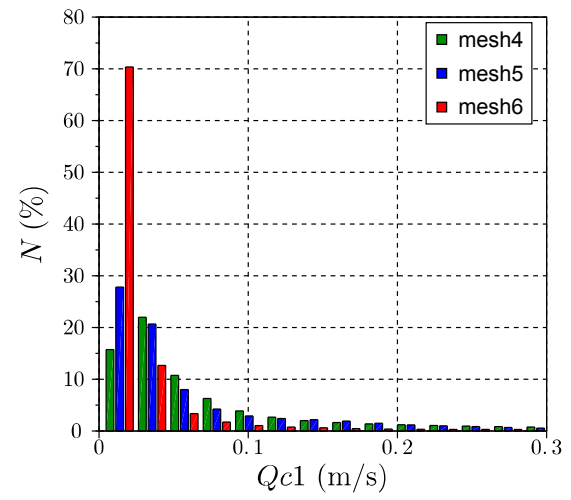

(a)

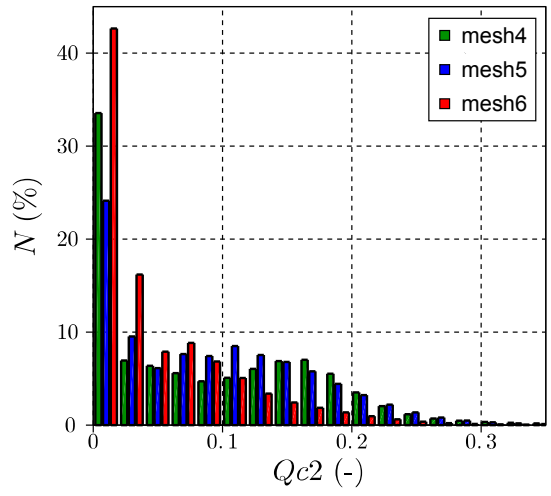

(b)

Fig. 20 Distribution of $Q c_{1}$ and $Q c_{2}$ for three meshes during the mesh adaptation steps; N (\%) represents the percentage of cells having a given criterion value in the mesh

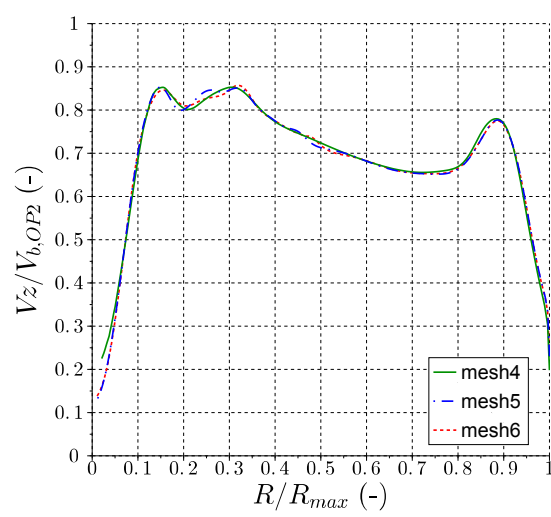

(a) Axial velocity

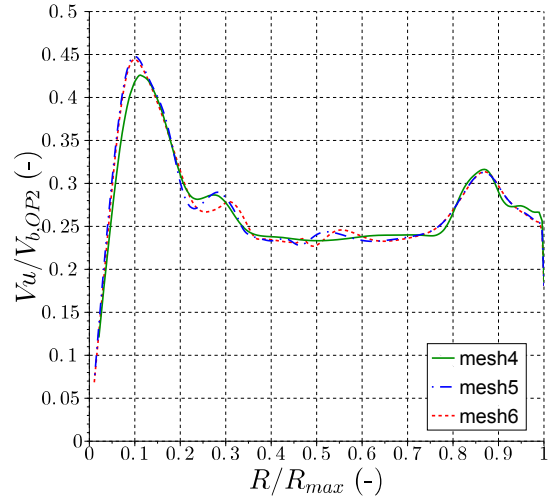

(b) Tangential velocity

Fig. 21 Non-dimensional axial (a) and tangential (b) velocity profiles (Vz, Vu) versus the non-dimensional radial position for OP2 for the meshes 4 to 6

3. A. Ruprecht, T. Helmrich, T. Aschenbrenner, T. Scherer, in Proceedings of 22nd IAHR Symposium on Hydraulic Machinery and Systems (2002), pp. 9-12

4. J. Paik, F. Sotiropoulos, M.J. Sale, Journal of hydraulic engineering 131(6), 441 (2005)

5. A. Gehrer, H. Benigni, M. Köstenberger, in 22nd IAHR Symposium on Hydraulic Machinery and Systems, Stockholm, vol. 29 (2004), vol. 29

6. P. Spalart, M. Shur, Aerospace Science and Technology 1(5), 297 (1997)

7. P.E. Smirnov, F.R. Menter, Journal of Turbomachinery 131(4), 041010 (2009)

8. M. Kato, B. Launder, in Ninth Symposium on Turbulent Shear Flows, Kyoto, Japan, August 16-18 (1993)

9. D. Jošt, A. Škerlavaj, A. Lipej, in IOP Conference Series: Earth and Environmental Science, vol. 15 (IOP Publishing, 2012), vol. 15, p. 062016 
10. D. Jošt, A. Škerlavaj, in IOP Conference Series: Earth and Environmental Science, vol. 22 (IOP Publishing, 2014), vol. 22, p. 022007

11. P. Bélanger-Vincent, Simulations avancées de l'écoulement turbulent dans les aspirateurs de turbines hydrauliques. Ph.D. thesis, Université Laval (2010)

12. C.A. Beaubien, Simulations numériques de l'écoulement turbulent dans un aspirateur de turbine hydraulique. Ph.D. thesis, Université Laval (2013)

13. C. Duprat, Simulation numérique instationnaire des écoulements turbulent dans les diffuseur de centrales hydrauliques en vue de l'amélioration des performances. Ph.D. thesis, Institut National Polytechnique de Grenoble-INPG (2010)

14. J.M. Gagnon, F. Flemming, R. Qian, C. Deschenes, S. Coulson, in ASME 2010 3rd Joint US-European Fluids Engineering Summer Meeting collocated with 8th International Conference on Nanochannels, Microchannels, and Minichannels (American Society of Mechanical Engineers, 2010), pp. 2227-2236

15. V. De Henau, F. Payette, M. Sabourin, C. Deschênes, J. Gagnon, P. Gouin, in IOP Conference Series: Earth and Environmental Science, vol. 12 (IOP Publishing, 2010), vol. 12, p. 012084

16. S. Houde, A. Carrier, J. Buron, C. Deschênes, in IOP Conference Series: Earth and Environmental Science, vol. 22 (IOP Publishing, 2014), vol. 22, p. 022009

17. F.A. Payette, Simulation de l'écoulement turbulent dans les aspirateurs de turbines hydrauliques: Impact des paramètres de modélisation. Ph.D. thesis, Université Laval (2008)

18. O. Brugière, Fiabilité et évaluation des incertitudes pour la simulation numérique de la turbulence: application aux machines hydrauliques. Ph.D. thesis, Grenoble University (2015)

19. H. Nilsson, M. Cervantes, in IOP Conference Series: Earth and Environmental Science, vol. 15 (IOP Publishing, 2012), vol. 15, p. 032002

20. E.R. Ortiz, J.M. Gagnon, C. Deschênes, in 18th Annual Conference CFD Society of Canada, CFD $2010(2010)$

21. F.R. Menter, NASA STI/Recon Technical Report N 93, 22809 (1992)

22. D. Marjavaara, R. Kamakoti, S. Lundström, S. Thakur, J. Wright, W. Shyy, in Turbine99 III: Proceedings of the third IAHR/ERCOFTAC workshop on draft tube flow (Luleå: Luleå tekniska universitet, 2005)

23. H. Keck, M. Sick, Acta Mechanica 201(1-4), 211 (2008)

24. J. Vuillemard, V. Aeschlimann, R. Fraser, S. Lemay, C. Deschênes, in IOP Conference Series: Earth and Environmental Science, vol. 22 (IOP Publishing, 2014), vol. 22, p. 032010

25. B.E. Launder, D. Spalding, Computer methods in applied mechanics and engineering $\mathbf{3}(2)$, $269(1974)$

26. V. Moureau, P. Domingo, L. Vervisch, Comptes Rendus Mécanique 339(2), 141 (2011)

27. M. Germano, U. Piomelli, P. Moin, W.H. Cabot, Physics of Fluids A: Fluid Dynamics (1989-1993) 3(7), 1760 (1991)

28. B. Mulu, P. Jonsson, M. Cervantes, Applied Energy 93, 695 (2012)

29. P. Jonsson, B. Mulu, M. Cervantes, Applied Energy 94, 71 (2012)

30. S. Lemay, Étude expérimentale de l'écoulement dans le canal inter-aube d'une turbine de type bulbe. Ph.D. thesis, Université Laval (2014)

31. M. Lesieur, O. Métais, P. Comte, Large-eddy simulations of turbulence (Cambridge University Press, 2005)

32. J. Ligen, M. Le Guay, Pertes de charge (Les Ãlditions parisiennes (EDIPA), 2007)

33. S.B. Pope, Turbulent flows (Cambridge university press, 2000)

34. G. MacIsaac, S. Sjolander, T. Praisner, Journal of Turbomachinery 134(6), 061015 (2012)

35. P.T. J. Cui, V. Nagabhushana Rao, in Eight International Symposium on Turbulence, Heat and Mass Transfer (Edited by K. Hanjalic, T. Miyauchi, D. Borello, M. Hadziabdic and P. Venturini) (2015)

36. U. Piomelli, W.H. Cabot, P. Moin, S. Lee, Physics of Fluids A: Fluid Dynamics (1989-1993) 3(7), 1766 (1991)

37. F. Menter, Y. Egorov, Flow, Turbulence and Combustion 85(1), 113 (2010)

38. A. Escue, J. Cui, Applied Mathematical Modelling 34(10), 2840 (2010)

39. C. Duprat, G. Balarac, O. Métais, P.M. Congedo, O. Brugière, Physics of Fluids (1994present) 23(1), 015101 (2011)

40. P. Benard, G. Balarac, V. Moureau, C. Dobrzynski, G. Lartigue, Y. D'Angelo, International Journal for Numerical Methods in Fluids (2015) 